\title{
Education needed - for doctors and patients - to reduce inappropriate antibiotic prescriptions
}

Cite as: CMAJ 2019 May 6:191:E514-5. doi: 10.1503/cmaj.109-5742

Posted on cmajnews.com on Apr. 18, 2019.

$\mathrm{T}$ he Chief Medical Officer of England recently warned that onethird of antibiotics prescribed in the National Health Service aren't prescribed for an appropriate illness. This is a reasonable estimate for Canada and the United States as well, according to Dr. Michael Silverman, chair of infectious diseases at Western University. Research in this area, overall, indicates that $20 \%-$ $40 \%$ of antibiotics are prescribed inappropriately, which increases the risk of antimicrobial resistance.

"Multi-drug-resistant organisms are something that are a major concern in Canada," Silverman said. "They're a major concern around the world."

The Government of Canada sees antimicrobial resistance as a serious and growing health threat, acknowledging that a "post-antibiotic era where common infections and minor injuries are once again deadly is a very real possibility for the 21st century."

In the United States, a recent study found that $23 \%$ of antibiotic prescriptions filled by outpatients were inappropriate, $36 \%$ were potentially appropriate, and $29 \%$ were not associated with a recent diagnosis code. A separate study found that about $40 \%$ of antibiotics filled by older adults in the US were potentially inappropriate.

Silverman has carried out similar research in Ontario. In a
2017 study he led, $46 \%$ of older adults received antibiotic prescriptions for nonbacterial acute upper respiratory infections. His research group is planning a similar study looking at prescription patterns in children and younger adults.

Silverman emphasized that physicians who overprescribe aren't trying to do harm. They're following what they believe is the standard of care. Prescription practices might depend on when and where physicians were trained.
Silverman's data showed that mid- and late-career physicians and physicians who were trained outside of Canada or the United States were more likely to prescribe antibiotics for nonbacterial infections.

Physicians who have been in the profession for longer may have been educated at a time when there was less awareness of the potential harms of prescribing antibiotics inappropriately, in terms of resistance and patient complications.

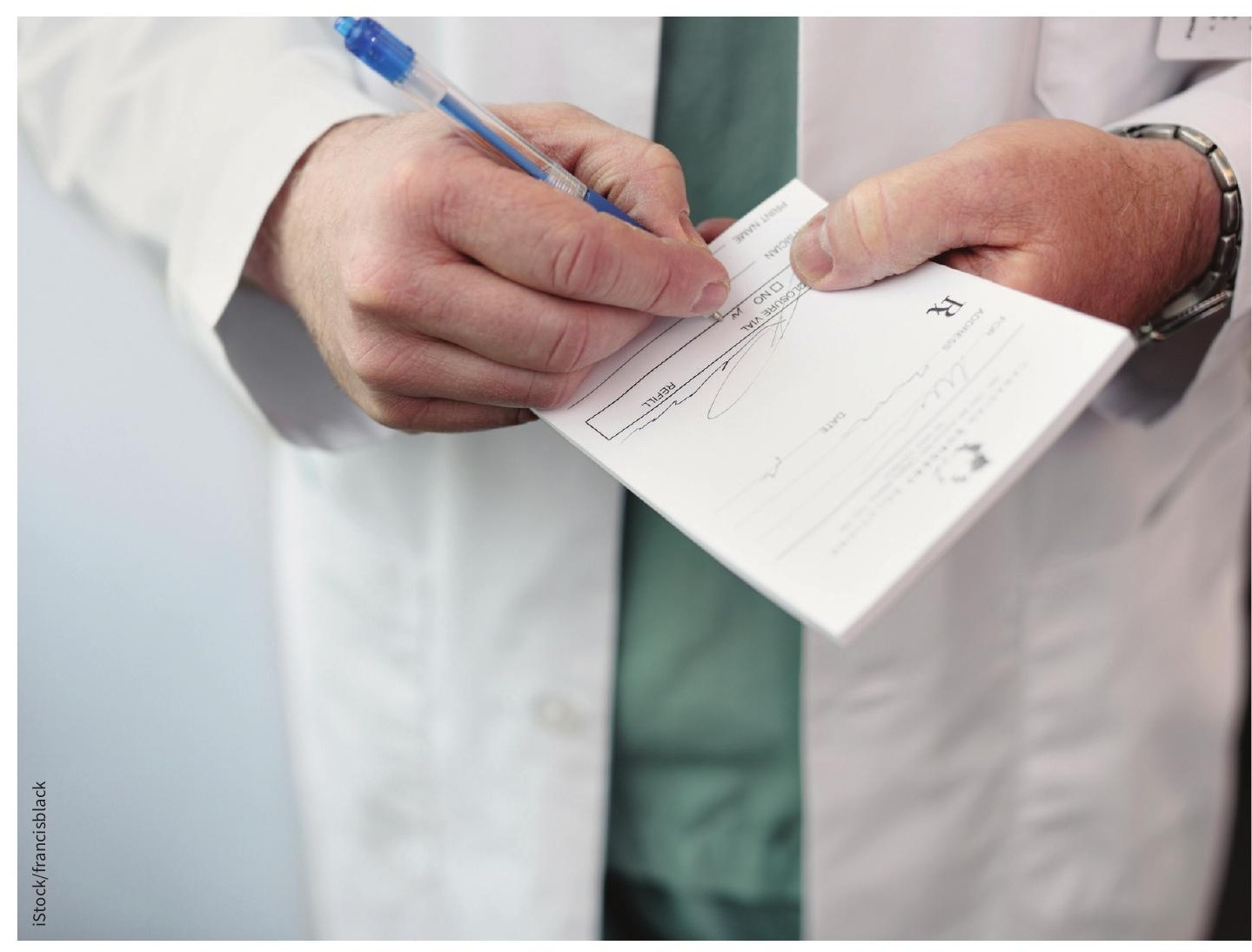

Research indicates that $20 \%-40 \%$ of antibiotics are prescribed inappropriately. 
Silverman said those physicians, in the past, would have been more likely to prescribe antibiotics "to be on the safe side" in cases of diagnostic uncertainty, a practice which could still be the norm for some.

Another problem is that physicians are often pressured to write prescriptions, especially when patients are ill and desperate for something to help them feel better. "It's a difficult thing to not write a script," Silverman said. "It takes a lot of emotional, intellectual energy."

It also takes more time to explain to patients why they don't need a prescription. In Silverman's study, physicians with higher patient volumes were more likely to write inappropriate prescriptions.

To help address this problem, Choosing Wisely Canada designed "viral prescription pads" with a written explana- tion of why a patient wasn't prescribed antibiotics, along with instructions to rest, drink fluids and take over-thecounter medication as suggested.

Dr. Alex Singer, a family physician and clinical champion for Choosing Wisely in Manitoba, said this is a good solution because it gives patients direction and reminds them that they'll probably feel better in a few days. Choosing Wisely also created a "delayed prescription" for patients whose symptoms might not resolve on their own.

In recent research that established a baseline for several Choosing Wisely recommendations, Singer found that $29 \%$ of the Manitoba-based providers involved in the study prescribed antibiotics to a patient with a viral infection, with prescription patterns differing between practices.
"This is a shared problem that we need to address as a profession," Singer said.

Both Singer and Silverman pointed out that shortening the duration of prescriptions is another way to reduce the threat of antibiotic resistance. A recent UK study found that a large proportion of antibiotics prescribed for common infections exceeded guidelines for duration of treatment. Together, the patients in the study were prescribed 1.3 million days of unnecessary antibiotics.

Silverman is hopeful that physician and patient education could lead to changes in the way antibiotics are prescribed. "It's a critical issue for the long-term sustainability of our health care system," he said. "We need antibiotics to work for just about everything we do in health care."

Caroline Mercer, Toronto, Ont. 